\title{
Streptococcus thermophilus ST28 Ameliorates Colitis in Mice Partially by Suppression of Inflammatory Th17 Cells
}

\author{
Tasuku Ogita, ${ }^{1}$ Megumi Nakashima, ${ }^{1}$ Hidetoshi Morita, ${ }^{2}$ Yasuo Saito, ${ }^{3}$ \\ Takuya Suzuki, ${ }^{1}$ and Soichi Tanabe ${ }^{1}$ \\ ${ }^{1}$ Graduate School of Biosphere Science, Hiroshima University, 1-4-4 Kagamiyama, Higashi-hiroshima, Hiroshima 739-8528, Japan \\ ${ }^{2}$ School of Veterinary Medicine, Azabu University, 1-17-71 Fuchinobe, Chuo, Sagamihara, Kanagawa 252-5201, Japan \\ ${ }^{3}$ Institute for Technical Research, Glico Dairy Products Co. Ltd., 2-14-1 Musashino, Akishima, Tokyo 196-0021, Japan
}

Correspondence should be addressed to Soichi Tanabe, stanabe@hiroshima-u.ac.jp

Received 30 December 2010; Revised 5 April 2011; Accepted 28 June 2011

Academic Editor: Dennis P. Murr

Copyright (c) 2011 Tasuku Ogita et al. This is an open access article distributed under the Creative Commons Attribution License, which permits unrestricted use, distribution, and reproduction in any medium, provided the original work is properly cited.

\begin{abstract}
The effects of Streptococcus thermophilus ST28 on cytokine production by murine splenocytes stimulated with transforming growth factor- $\beta$ plus interleukin- (IL-) 6 were evaluated. The addition of ST2 8 significantly repressed IL-17 production compared to ATCC 19258 (type strain). ST28 also decreased the number of Th17 cells in the stimulated splenocytes. The anti-inflammatory effects of ST28 administration were evaluated in mice with colitis induced by dextran sodium sulphate (DSS). Oral treatment of mice with ST28 ameliorated the intestinal lesions by DSS. Upon DSS treatment, IL-17 production in lamina propria lymphocytes (LPLs) was induced, but ST28 significantly decreased its production. ST28 also decreased the percentage of Th17 cells in LPL from DSSinduced colitis. The present results imply that ST28 suppresses the Th17 response in inflamed intestines and would be useful in the treatment of Th17-mediated diseases, such as inflammatory bowel disease.
\end{abstract}

\section{Introduction}

A number of studies have documented the potential benefits of probiotic bacteria which prevent and ameliorate human T-helper-2-cell- (Th2-) related diseases, such as atopic dermatitis and asthma [1]. At the helper T-cell level, a number of probiotic lactobacilli promote the production of T-helper1-cel-1 (Th1-) skewing cytokines, such as interleukin (IL-) 12 and interferon (IFN-) $\gamma$, and suppress the production of IgE and Th2 cytokines [2]. Other studies have demonstrated that bifidobacteria suppress Th2 cytokine and IgE production, without significant induction of Th1 cytokines [3]. The Th2-suppressive effect of probiotic bacteria is mediated by regulatory $\mathrm{T}$ (Treg) cells linked to the induction of regulatory cytokines, including IL-10 and transforming growth factor (TGF-) $\beta[4,5]$. It has been reported that macrophages stimulated with Lactococcus lactis W58 [4] produce IL-10 and that TGF- $\beta$ is induced by Lactobacillus paracasei NCC246 in mixed lymphocyte reactions [5].

In addition, recent clinical studies have proven the prevention and curative features of probiotics in some intestinal disorders, such as inflammatory bowel diseases (IBD) including Crohn's disease and ulcerative colitis. It was demonstrated that administration of Bifidobacterium longum BB536 was effective in inducing remission of patients with ulcerative colitis [6]. Multiple mechanisms of action have been suggested to explain the protective effects of probiotics in intestinal inflammation. These can be broadly classified as follows: (1) suppression of growth or epithelial binding/invasion by pathogenic bacteria, (2) improved epithelial barrier function, and (3) immunoregulatory activities [7]. As for immunoregulation, several probiotics can induce protective cytokines, including IL-10 and TGF- $\beta$, and suppress pro-inflammatory cytokines, such as TNF- $\alpha$, in the mucosa of patients with paucities and Crohn's disease [8].

Besides Th1, Th2, and Treg, Thelper 17 cells (Th17) have received considerable recent attention as they exhibit effector functions distinct from Th1 and Th2 cells $[9,10]$. Differentiation factors (TGF- $\beta$ plus IL-6 or IL-21), growth and stabilization factor (IL-23), and transcription factors (signal transducer and activator transcription 3 (STAT3), retinoic acid receptor-related orphan receptor (ROR-) $\gamma t$ and 
ROR- $\alpha$ ) involved in the development of Th17 cells have been identified [10].

Notably, Th17 cells produce the pleiotropic cytokine IL17 , which potently induces tissue inflammation and is associated with the pathogenesis of many human diseases, such as IBD [11] and rheumatoid arthritis [12]. Thus, modulation of Th17 cells is currently viewed as a potentially positive pharmacological outcome. Although some studies have revealed that lactic acid bacteria and bifidobacteria may have potential use as anti-inflammatory agents in certain chronic inflammatory diseases, such as IBD [13], it is unclear whether these bacteria are able to suppress excess Th17 activity.

We recently reported that Bifidobacterium longum infantis JCM 1222 (type strain) suppresses IL-17 production in murine splenocytes in vitro and in inflamed intestinal cultures ex vivo [14]. To identify other bacterial strains with potential Th17 modulating activity, we previously performed an in vitro assay with 200 strains of streptococci and found that Streptococcus thermophilus ST28 exhibited potent inhibitory activity towards IL-17 production [15]. The main objective of this study was to evaluate the effects of oral administration of ST28 on IL-17 production by murine lamina propria lymphocytes (LPLs) and on the expression of Th17-related surface markers in inflamed intestines. We first evaluated and compared in vitro cytokine production patterns in Th17-skewed conditions following the exposure of splenocytes to ST28 and ATCC 19258 (type strain). The in vivo effects of ST28 were also evaluated using flow cytometry and real-time PCR in dextran-sodium-sulphate(DSS-) induced colitis mice, a human IBD model in which IL-17 plays a pivotal role in pathogenesis [16].

\section{Materials and Methods}

2.1. Bacterial Strains. Two strains of $S$. thermophilus, ATCC 19258 (type strain) and ST28, were obtained from the American Type Culture Collection and Glico Dairy Products (Tokyo, Japan), respectively. Each strain was cultured in M17 broth (Merck, Whitehouse Station, NJ, USA) at $37^{\circ} \mathrm{C}$ for $17-$ 65 hours. The cultured cells were washed with sterile distilled water and incubated at $100^{\circ} \mathrm{C}$ for $50 \mathrm{~min}$ [15]. Following the incubation, the heat-killed bacteria were lyophilized and used for subsequent experiments.

2.2. Suppressive Effect of $S$. thermophilus ST28 and ATCC 19258 on In Vitro IL-17 Production in Murine Splenocytes. Six-week-old female Balb/c mice were obtained from Charles River Japan (Kanagawa, Japan), and all experimental protocols involving animals were approved by the Animal Care Committee, Graduate School of Biosphere Science, Hiroshima University. To harvest splenocytes, mice were sacrificed by cervical dislocation, spleens were removed, and suspensions of splenocytes $\left(1.2 \times 10^{6}\right.$ cells $)$ were incubated with $2 \mathrm{ng} / \mathrm{mL}$ TGF- $\beta$ (R\&D Systems, Minneapolis, Minn, USA) plus $20 \mathrm{ng} / \mathrm{mL}$ IL-6 (R\&D Systems) at $37^{\circ} \mathrm{C}$ for 72 hours in $120 \mu \mathrm{l}$ RPMI 1640 medium (Life Technologies, Foster City, California, USA) supplemented with $10 \%$ fetal bovine serum (FBS, ICN Biomedicals, Osaka, Japan), $10 \mu \mathrm{M}$ 2-mercaptoethanol, $10 \mathrm{mM}$ HEPES, 5 units/mL penicillin (Life Technologies), and $5 \mu \mathrm{g} / \mathrm{mL}$ streptomycin (Life Technologies) in a 96-well cell culture plate (Thermo Fisher Scientific, Waltham, MA, USA). Heat-killed bacteria $(1.2 \times$ $10^{6}$ cells) were then added to the cell cultures. A culture to which no TGF- $\beta$ plus IL- 6 or heat-killed bacterial cells were added was included as a control. Following the incubation, the plate was centrifuged. Cells were applied to flow cytometry (Figure 1), and culture supernatants were assayed for cytokine concentrations (Table 1) as described below.

2.3. Flow Cytometric Analysis of Splenocytes. The splenocytes $\left(1 \times 10^{6}\right.$ cells $\left./ \mathrm{mL}\right)$ were incubated at $37^{\circ} \mathrm{C}$ for 4 hours in RPMI 1640 medium containing $0.5 \mu \mathrm{g} / \mathrm{mL}$ phorbol12-myristate-13-acetate (MP Biomedicals, Aurora, Ohio, USA), $1 \mu \mathrm{g} / \mathrm{mL}$ ionomycin (Wako Pure Chemical Industries, Osaka, Japan), and $3 \mu \mathrm{g} / \mathrm{mL}$ brefeldin-A (Wako Pure Chemical Industries). Then, anti-mouse CD32/16 antibody was added and incubated $4^{\circ} \mathrm{C}$ for $30 \mathrm{~min}$. For analysis of surface markers, fluorescein isothiocyanate (FITC) antimouse CD11c antibody (BioLegend, San Diego, Calif, USA), allophycocyanin (APC) anti-mouse CD11b antibody (eBioscience, San Diego, Calif, USA), or APC anti-mouse CD4 antibody (eBioscience) was added to the cell suspension, which was then incubated in the dark at $4^{\circ} \mathrm{C}$ for $30 \mathrm{~min}$. For isotype controls, FITC-Armenian hamster IgG, APC rat IgG2a $\kappa$ (BioLegend), or APC Rat IgG2b $\kappa$ (eBioscience) was used. After the incubation, the cells were permeabilized by IntraPrep (Beckman Coulter, Marseille codex, France) for intracellular cytokine staining. Phycoerythrin (PE) antimouse IL-17 antibody (eBioscience) or PE rat IgG2a $\kappa$ (eBioscience) was added to the cell suspension and incubated in the dark at $4^{\circ} \mathrm{C}$ for $30 \mathrm{~min}$. Following the incubation, the cells were washed twice with 5\% FBS-Hanks' balanced salt solution (HBSS) and suspended in $300 \mu \mathrm{L}$ of $5 \%$ FBS-HBSS. Fluorescence intensity was measured on a Guava Easycyte flow cytometry system (Millipore, Billerica, Mass, USA).

2.4. Cytokine Measurements. Cytokine (IFN- $\gamma$, IL-2, IL4, IL-5, IL-10, IL-12(p70), tumor-necrosis-factor- (TNF-) $\alpha$, and granulocyte macrophage colony-stimulating factor (GM-CSF)) concentrations of the culture supernatants were determined by the microbead method using the Bio-Plex Suspension Array System (BioRad Laboratories, Hercules, Calif, USA), according to the manufacturer's instructions [17]. Briefly, culture supernatants were incubated with beads conjugated with anti-IFN- $\gamma$, -IL-2, -IL-4, -IL-5, -IL-10, -IL-12(p70), -TNF- $\alpha$, and -GM-CSF antibodies (BioRad Laboratories) followed by sandwich immunoassay using biotinylated secondary antibodies. The beads were washed three times after each incubation, and PE-streptavidin was used as a reporter. The relative fluorescence units were determined by counting 100 beads using the Bio-Plex system. Data were evaluated with the Bio-Plex Manager Software 6.0 (BioRad Laboratories). Separately, the IL-17 concentration of culture supernatants was measured using a DuoSet Sandwich ELISA Kit (R\&D Systems) following the manufacturer's instructions. 
TABLE 1: Changes in cytokine production patterns by murine splenocytes in Th17-skewed conditions by the addition of $S$. thermophilus ST28 or S. thermophilus ATCC 19258.

\begin{tabular}{|c|c|c|c|c|}
\hline \multirow{2}{*}{ Cytokine (pg/mL) } & \multirow{2}{*}{ Control } & \multirow[b]{2}{*}{$(-)$} & \multicolumn{2}{|c|}{ TGF- $\beta+$ IL-6 (+) } \\
\hline & & & S. thermophilus ST28 & S. thermophilus ATCC 19258 \\
\hline IL-17 & $412 \pm 17^{* *}$ & $4,383 \pm 192$ & $976 \pm 21^{* * \# \#}$ & $4,858 \pm 116$ \\
\hline IFN- $\gamma$ & $616 \pm 47^{* *}$ & $4,960 \pm 105$ & $2,215 \pm 369^{* * \# \#}$ & $564 \pm 91^{* *}$ \\
\hline IL-2 & $909 \pm 325^{* *}$ & $102 \pm 7$ & $1,311 \pm 344^{* * \# \#}$ & $9,384 \pm 96$ \\
\hline IL-4 & $588 \pm 30^{* *}$ & n.d. & $135 \pm 27^{* * \# \#}$ & $297 \pm 19^{* *}$ \\
\hline IL-5 & $453 \pm 112^{*}$ & $8 \pm 4$ & $61 \pm 21$ & $115 \pm 9$ \\
\hline IL-10 & $1,348 \pm 163^{* *}$ & $27,251 \pm 2,594$ & $2,550 \pm 253^{* *}$ & $2,773 \pm 62^{* *}$ \\
\hline IL-12(p70) & $508 \pm 64^{* *}$ & $10,778 \pm 1,029$ & $985 \pm 100^{* *}$ & $1,073 \pm 25^{* *}$ \\
\hline TNF- $\alpha$ & $6,259 \pm 505^{* *}$ & $52,511 \pm 1,123$ & $23,282 \pm 3,927^{* * \# \#}$ & $5,696 \pm 971^{* *}$ \\
\hline GM-CSF & $125 \pm 75^{* *}$ & $12,070 \pm 1,196$ & $679 \pm 117^{* *}$ & $782 \pm 29^{* *}$ \\
\hline
\end{tabular}

n.d., not detected. ${ }^{*} P<0.01,{ }^{*} P<0.05\left((-)\right.$ versus Control, $S$. thermophilus ST28, or S. thermophilus ATCC 19258) ${ }^{\#} P<0.01$ (S. thermophilus ST28 versus S. thermophilus ATCC 19258).

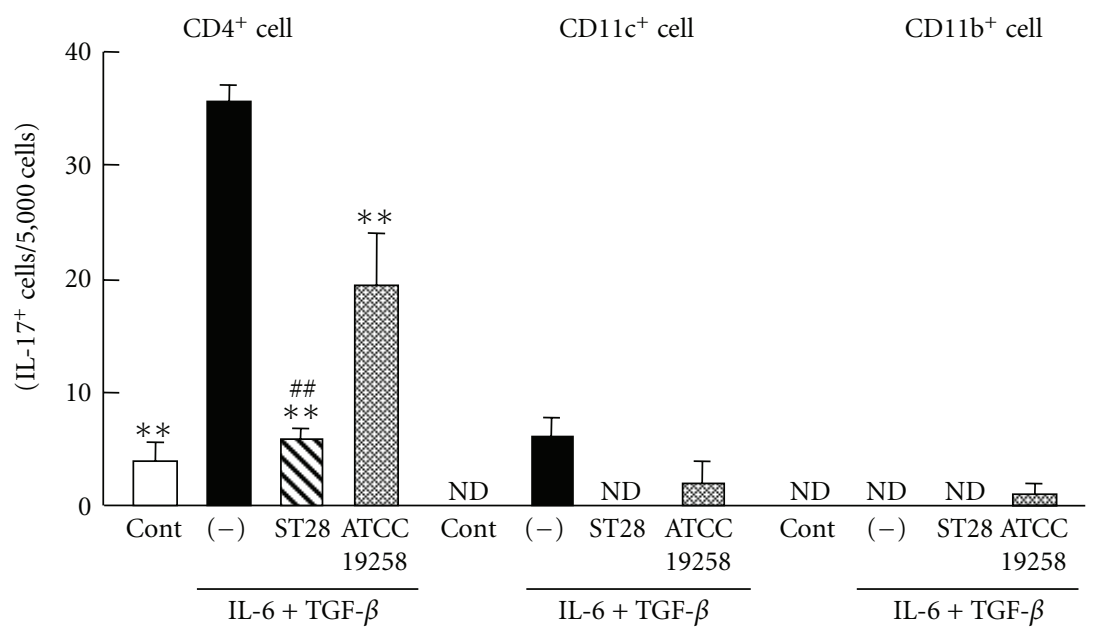

FIGURE 1: The suppressive effect of $S$. thermophilus ST28 on Th17 cells in murine splenocytes. Splenocytes were stimulated with TGF- $\beta$ plus

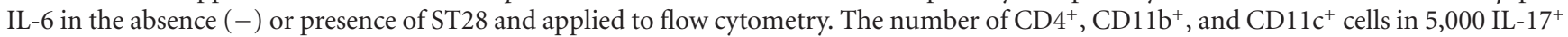
cells is indicated. Splenocytes without the stimulation of TGF- $\beta$ plus IL-6 were used as controls (Cont). \# $P<0.01$ (S. thermophilus ST28 versus S. thermophilus ATCC 19258). ${ }^{* *} P<0.01$ ((-) versus Cont, S. thermophilus ST28, or S. thermophilus ATCC 19258). ND, not detected.

2.5. Administration of S. thermophilus ST28 or ATCC 19258 to DSS-Induced Colitis Mice. Six-week-old female BALB/c mice were housed in an air-conditioned room under a 12 hours light-dark cycle and were allowed ad libitum access to tap water and a standard diet (MF; Oriental Yeast, Tokyo, Japan). Acute colitis was induced in mice by adding 3.5\% (w/v) DSS (molecular weight, 36,000-50,000; MP Biomedicals) to their drinking water for 5 days [18]. The experimental protocols (Figure 1(a)) were approved by the Animal Care Committee, Graduate School of Biosphere Science, Hiroshima University.

Animals were randomly divided into four groups $(n=$ 4 each). Group 1 (control group) mice were treated orally with phosphate-buffered saline (PBS) from days 1 to 3 , while Group 2 (control group) received drinking water containing $3.5 \%(\mathrm{w} / \mathrm{v})$ DSS and were orally administered PBS. Groups 3 and 4 also received drinking water containing 3.5\% (w/v) DSS, and, 24 hours after the start of DSS administration, the mice were orally administered $10^{7} \mathrm{cfu} /$ day of heat-killed ST28 (group 3) or ATCC 19258 (group 4) in PBS for 3 consecutive days (Figure 2(a)). The severity of intestinal inflammation was assessed on day 5 by assigning a score for each of the 2 stool scores, consistency, and bleeding (Figure 2(b)). The scale used for consistency was 0 (normal stool), 1 (loose stool), 2 (diarrheal stool), and 3 (watery stool), and that used for bleeding was 0 (normally colored stool), 1 (blood visibly present in the stool), 2 (adherent of blood on anus), and 3 (gross bleeding on anus). Then, the total score was expressed as the sum of the scores of the 2 items (total score, 6). After the experimental period, the entire colon was removed from mice (Figure 2(c)), and lamina propria from the excised colons was removed.

2.6. Preparation of Lamina Propria Lymphocytes (LPLs). Pooled $(n=4)$ colon tissue was suspended in HBSS containing $10 \mathrm{mM}$ dithiothreitol (Wako Pure Chemical Industries) and incubated at $20^{\circ} \mathrm{C}$ for $10 \mathrm{~min}$ with shaking. The tissue was further treated with $2.1 \mathrm{mg} / \mathrm{mL}$ collagenase $\mathrm{D}$ (Wako 


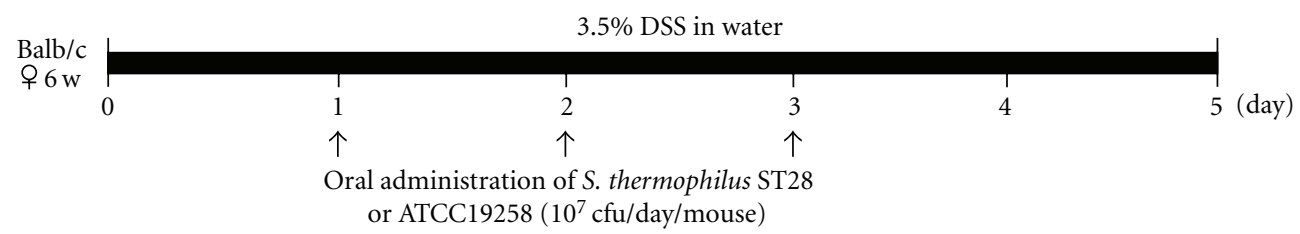

(a)

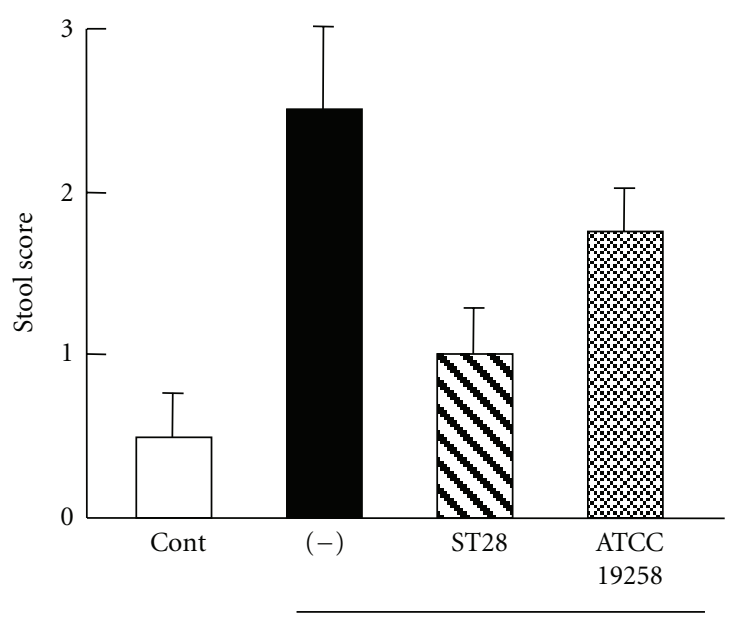

DSS

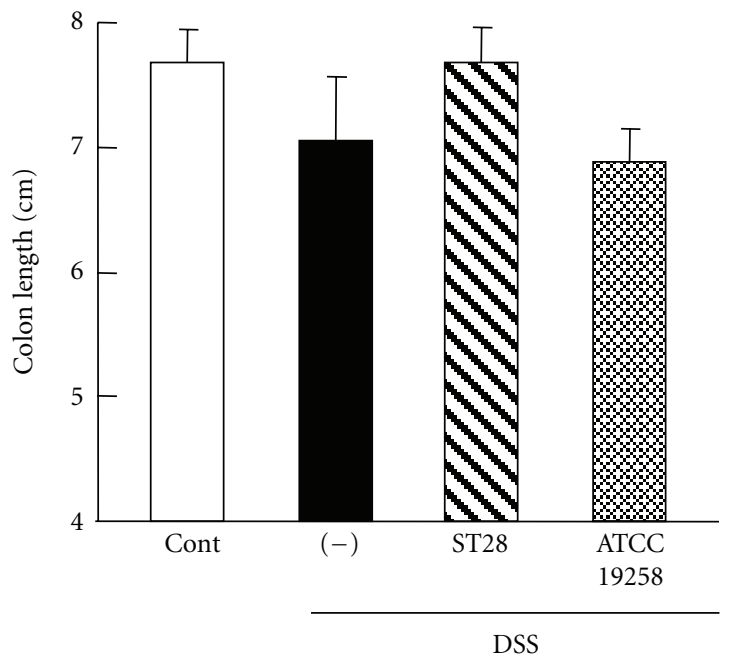

(c)

FIGURE 2: Effects of S. thermophilus ST28 administration on colons of dextran-sodium-sulphate- (DSS-) induced colitis mice. (a) Overview of the experimental design. Acute colitis was induced in mice $(n=4$ for each group) by exposure to $3.5 \%$ DSS for 5 days. On days $1-3$, mice were orally administered ST28 cells or ATCC 19258 ( $10^{7} \mathrm{cfu} /$ day). (b) Inflammation score on Day 5. The severity of inflammation was assessed by assigning a score for consistency and bleeding of stool (full score, 6). (c) Colon length on Day 5. Cont, healthy control mice, (-) untreated DSS-induced colitis mice.

Pure Chemical Industries) at $37^{\circ} \mathrm{C}$ for $20 \mathrm{~min}$ in a shaking water bath $[19,20]$. Only live cells were collected from single-cell suspensions from the LPLs by Dead Cell Removal kit (Miltenyi Biotec, Auburn, Calif, USA), and used for subsequent experiments.

2.7. Ex Vivo Culture of LPLs and Flow Cytometric Analysis. Portion of LPLs $\left(1 \times 10^{6}\right.$ cells $)$ were suspended in the same RPMI 1640 medium as described above and incubated at $37^{\circ} \mathrm{C}$ for 48 hours in the presence of $1 \mu \mathrm{g} / \mathrm{mL}$ antiCD3 (eBioscience) and $1 \mu \mathrm{g} / \mathrm{mL}$ anti-CD28 (eBioscience). Following the incubation, the plate was centrifuged. Cells were applied to flow cytometry, and culture supernatants were assayed for IL-17 and IFN- $\gamma$ concentrations (Figure 3).

For analysis of intracellular IL-17 (Figure 4(a)), LPLs $\left(1 \times 10^{6}\right.$ cells $\left./ \mathrm{mL}\right)$ were incubated at $37^{\circ} \mathrm{C}$ for 4 hours in RPMI 1640 medium containing $0.5 \mu \mathrm{g} / \mathrm{mL}$ phorbol12 -myristate-13-acetate, $1 \mu \mathrm{g} / \mathrm{mL}$ ionomycin, and $3 \mu \mathrm{g} / \mathrm{mL}$ brefeldin A. Then, APC anti-mouse CD4 antibody or APC rat $\operatorname{IgG} 2 \mathrm{a} \kappa$ was added to the cell suspension. After the incubation, the cells were permeabilized by IntraPrep, and $\mathrm{PE}$ anti-mouse IL-17 antibody or PE rat IgG2a $\kappa$ was added to the cell suspension. Following the incubation, the cells were applied to flow cytometry.

For analysis of surface markers (Figure 4(b)), freshly prepared LPLs were incubated with FITC anti-mouse CD11c antibody and APC anti-mouse CD86 antibody (eBioscience), or with FITC Armenian hamster IgG and APC rat IgG2a were added to the cell suspension and applied to flow cytometry.

2.8. mRNA Expression Analysis of LPLs. Pooled $(n=4)$ colon tissue from other DSS colitis mice was suspended in HBSS containing $5 \mathrm{mM}$ EDTA and incubated at $37^{\circ} \mathrm{C}$ for $20 \mathrm{~min}$ in a shaking water bath. The tissue was further treated with $1 \mathrm{mg} / \mathrm{mL}$ collagenase D (Roche Applied Science, Nonnenwald, Germany) and $3 \mathrm{mg} / \mathrm{mL}$ dispase II (Life Technologies) at $37^{\circ} \mathrm{C}$ for $30 \mathrm{~min}$ in a shaking water bath. After treatment, LPLs were purified by Percoll density-gradient centrifugation. Briefly, tissue was resuspended in $40 \%$ Percoll (GE Healthcare Biosciences AB, Uppsala, Sweden) and overlaid on $80 \%$ Percoll, after which density gradient was performed by centrifugation at $1,000 \mathrm{~g}$ for $20 \mathrm{~min}$ at $25^{\circ} \mathrm{C}$. LPLs were subsequently collected from the interface fraction between the $40 \%$ and $80 \%$ Percoll's layers $[19,20]$.

RNA was extracted from the LPLs using TRIzol (Life Technologies) following the manufacturer's instructions. Reverse transcription reactions were performed with HighCapacity cDNA Reverse Transcription Kit (Life Technologies) at $25^{\circ} \mathrm{C}$ for $10 \mathrm{~min}$ and $37^{\circ} \mathrm{C}$ for $120 \mathrm{~min}$. The reaction was terminated by heating at $85^{\circ} \mathrm{C}$ for $5 \mathrm{sec}$, followed by cooling at $4^{\circ} \mathrm{C}$. Real-time PCR was performed using 


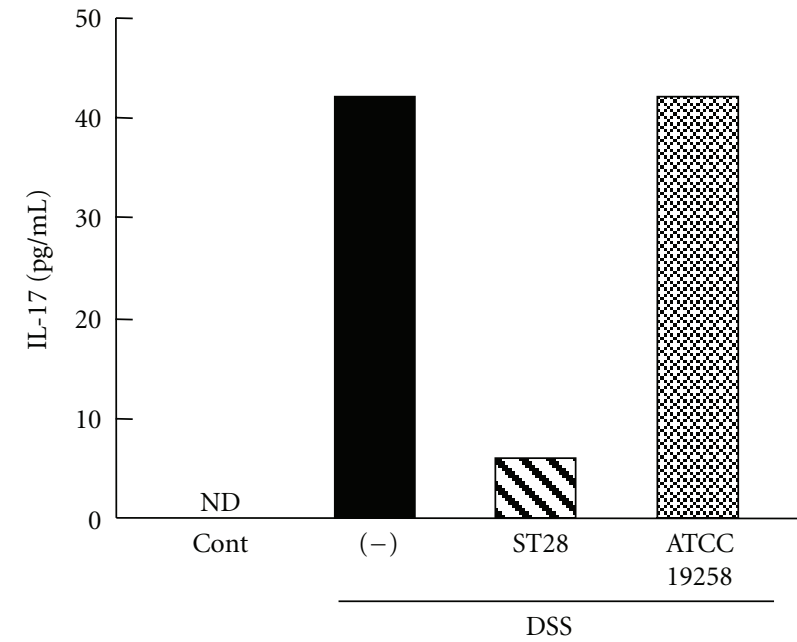

(a)

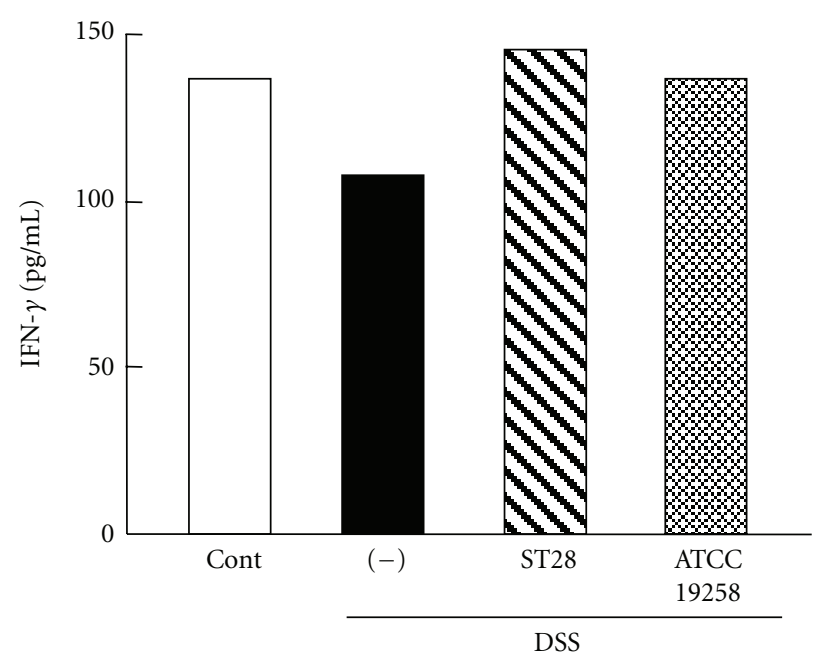

(b)

FIGURE 3: Effects of S. thermophilus ST28 administration on cytokine production in the lamina propria lymphocytes (LPLs) from DSSinduced colitis mice. LPLs from DSS-induced mice orally administered ST28 or ATCC 19258 were incubated with anti-CD3 and anti-CD28. Culture supernatants were harvested and assayed for concentrations of IL-17 (a) and IFN- $\gamma$ (b). LPLs from untreated DSS-induced colitis mice (-) and healthy control mice (Cont) were also assayed. ND, not detected.

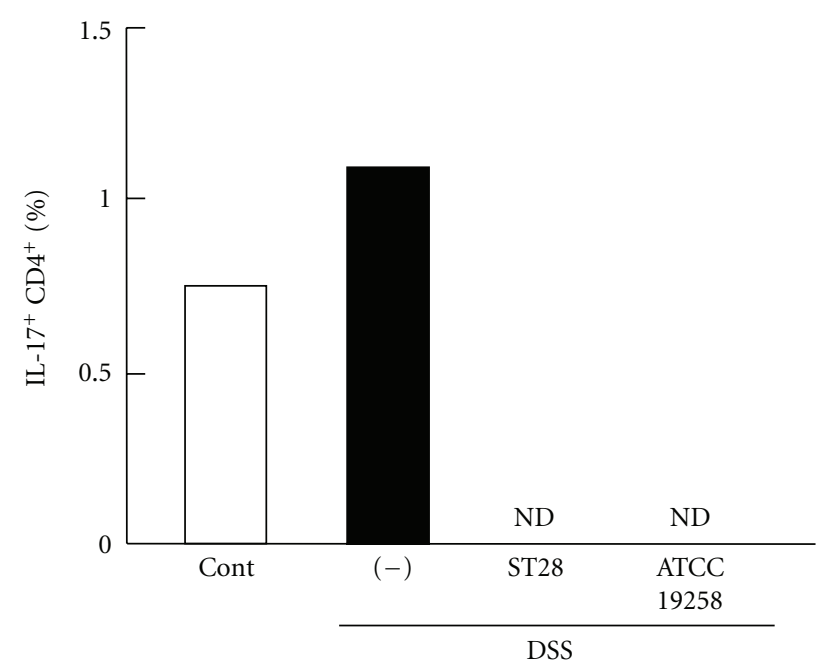

(a)

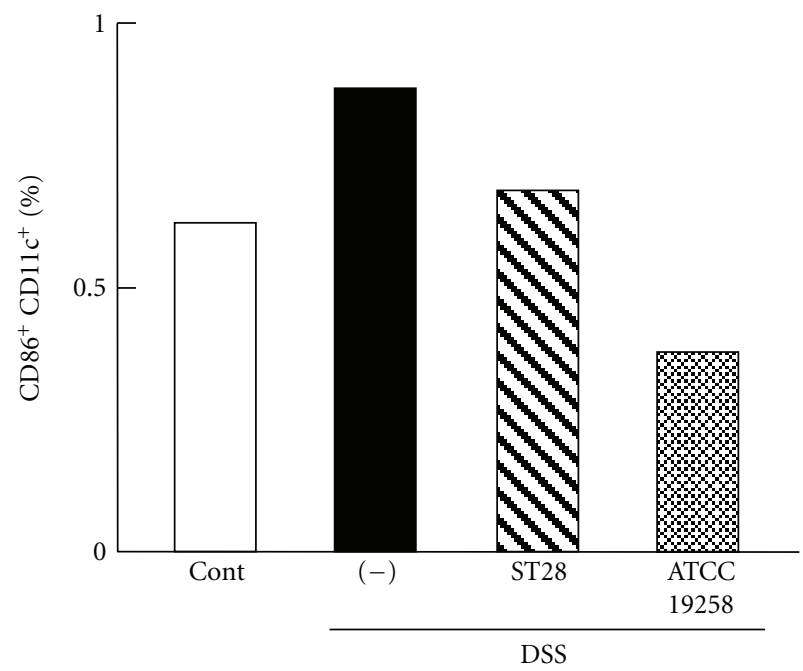

(b)

FIGURE 4: Effects of S. thermophilus ST28 on percentages of Th17 (a) and inflammatory DC (b) in LPLs from DSS-induced colitis mice. (a) After the experiments described in Figure 3, LPLs from DSS-induced mice orally administered ST28 or ATCC 19258 were incubated with phorbol-12-myristate-13-acetate, ionomycin, and brefeldin A and applied to flow cytometry to measure intracellular IL-17. (b) Separately, flow cytometric analysis of surface marker CD86 was performed using freshly prepared LPLs. LPLs from untreated DSS-induced colitis mice $(-)$ and healthy control mice (Cont) were also assayed. ND, not detected.

KAPA SYBR Fast ABI Prism qPCR Kit (Kapa Biosystems, Woburn, Mass, USA) and the primer sequences listed (/see/ in Supplementary Material available on line at doi: $10.1155 / 2011 / 378417)$. Reactions were performed at $95^{\circ} \mathrm{C}$ for $2 \mathrm{~min}$, followed by 40 cycles of $95^{\circ} \mathrm{C}$ for $5 \mathrm{sec}$ and $60^{\circ} \mathrm{C}$ for $30 \mathrm{sec}$. The dissociation stage was analyzed at $95^{\circ} \mathrm{C}$ for $15 \mathrm{sec}$, followed by 1 cycle of $60^{\circ} \mathrm{C}$ for $15 \mathrm{sec}$ and $95^{\circ} \mathrm{C}$ for $15 \mathrm{sec}$. The fluorescence of the SYBR green dye was determined as a function of the PCR cycle number, giving the threshold cycle number at which amplification reached a significant threshold. Data were analysed by the $\Delta \Delta \mathrm{Ct}$ method and presented as fold changes in gene expression after normalization to the internal control $\beta$-actin gene expression level (Supplementary Figure 1). generated according to the method of D'Arienzo et al. [21]. 


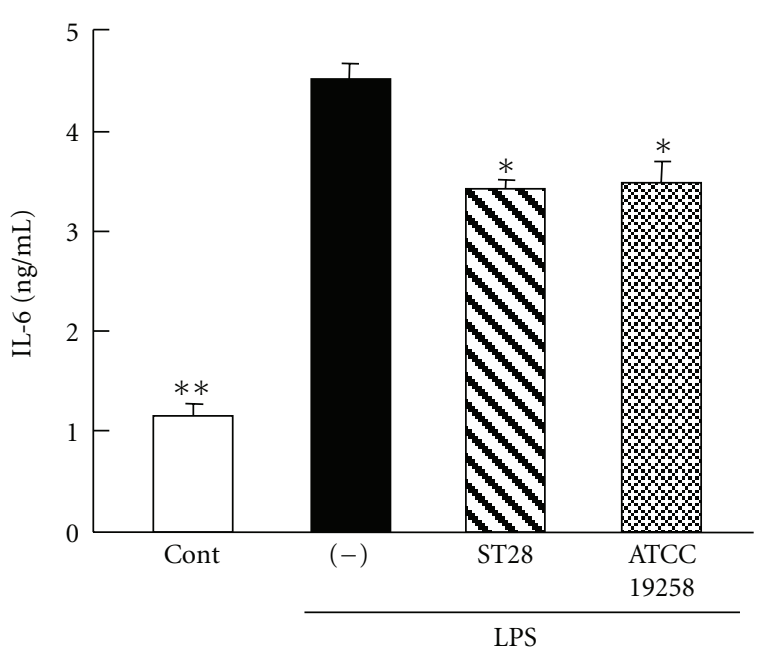

(a)

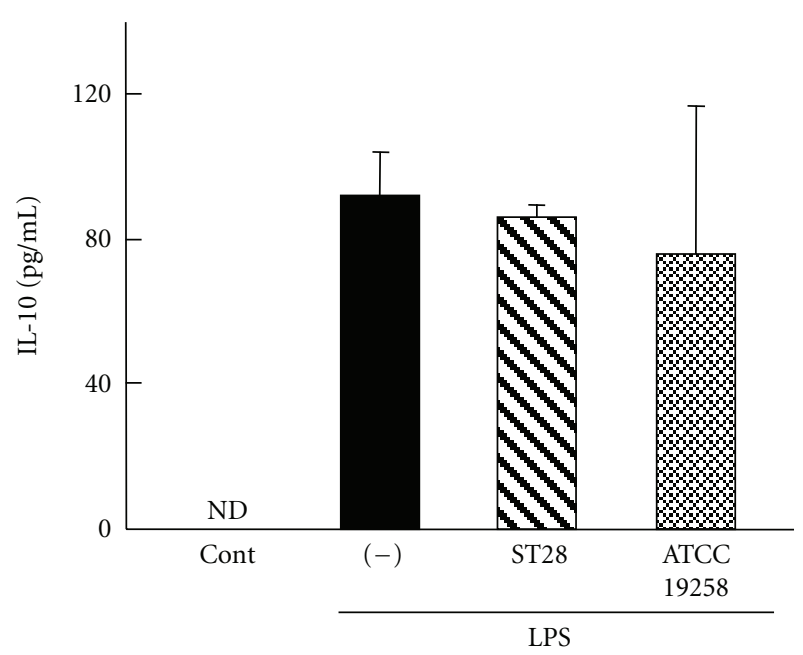

(b)

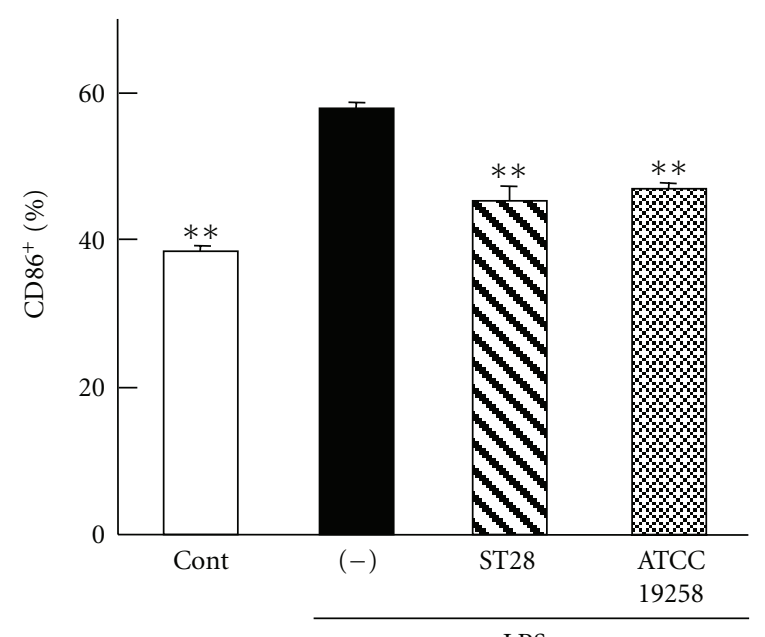

LPS

(c)

FIGURE 5: Effects of S. thermophilus ST28 on the production of IL-6 (a) and IL-10 (b) and the percentage of CD86 ${ }^{+}$cells (c) in bone marrow dendritic cells (BMDC). LPS-matured BMDC were incubated in the absence (-) or presence of ST28 or ATCC 19258 and assayed for concentrations of IL-6 (a) and IL-10 (b). CD86 $6^{+}$cells were analyzed by flow cytometry (c). BMDCs without LPS activation were used as controls (Cont). ${ }^{* *} P<0.01,{ }^{*} P<0.05$ ((-) versus Cont, S. thermophilus ST28, or S. thermophilus ATCC 19258). ND, not detected.

Briefly, bone marrow cells were collected from femurs of sixweek-old female Balb/c mice. Bone marrow mononuclear cells $\left(2 \times 10^{6}\right.$ cells $\left./ \mathrm{mL}\right)$ were cultured at $37^{\circ} \mathrm{C}$ for 7 days in RPMI1640 medium containing mouse 200 units/mL granulocyte macrophage colony-stimulating factor (Miltenyi Biotec), 10\% FBS, $10 \mu \mathrm{M}$ 2-mercaptoethanol, $10 \mathrm{mM}$ HEPES, 5 units $/ \mathrm{mL}$ penicillin, and $5 \mu \mathrm{g} / \mathrm{mL}$ streptomycin in a tissue culture flask. On day 7, BMDC $\left(1 \times 10^{6}\right.$ cells $\left./ \mathrm{mL}\right)$ were seeded in 24-well cell culture plate and matured by $1 \mu \mathrm{g} / \mathrm{mL}$ lipopolysaccharide (LPS, Sigma-Aldrich, St. Louis, Mo, USA) for 24 hours. On day 8, heat-killed bacteria (1 $\times 10^{7}$ cells/well) were added to the cell cultures. After 24hour incubation, the plate was centrifuged. IL- 6 and IL-10 concentrations of culture supernatants were measured using a DuoSet Sandwich ELISA Kit (R\&D Systems) following the manufacturer's instructions. CD86 ${ }^{+}$cells were analyzed by flow cytometry (Figure 5).
2.10. Data Analyses. Statistical analysis was performed using one-way ANOVA followed by Tukey's post hoc test. All data are expressed as the mean \pm standard error (S.E.).

\section{Results}

3.1. S. thermophilus ST28 Suppressed Th17 in Murine Splenocytes. When live ST28 was added to murine splenocytes and coincubated at $37^{\circ} \mathrm{C}$ for 72 hours, ST28 grew in RPMI 1640 medium, and it became impossible to properly examine the effect of ST28 on splenocytes. Thus, in subsequent experiments, ST28 and ATCC 19258 were used after heat killing.

Prior to the experiment, we first purified $\mathrm{T}$ cells from splenocytes by magnetic bead-based cell separation (MACS), and stimulated the MACS-purified T cells with TGF- $\beta$ plus IL-6. However, IL-17 production was not detectable (data not shown), which suggested that IL-17 production 
from Th17 cells in splenocytes required the coexistence of non-T cells. Therefore, we alternatively stimulated whole splenocytes with TGF- $\beta$ plus IL- 6 for further analyses, after which the stimulated splenocytes were applied to flow cytometry in order to analyze $\mathrm{CD} 4^{+}, \mathrm{CD} 1 \mathrm{~b}^{+}$, and $\mathrm{CD} 11 \mathrm{c}^{+}$ cells separately. As a result, most of the $\mathrm{IL}-17^{+}$cells were $\mathrm{CD}^{+} \mathrm{T}$ cells, which indicated that the major source of IL-17 was Th17 cells. More importantly, it was confirmed that ST28 decreased the percentage of $\mathrm{CD} 4^{+} \mathrm{IL}-17^{+}$cells (Figure 1).

\subsection{S. thermophilus ST28 Modulated In Vitro Cytokine Pro-} duction in Th17-Skewed Conditions. Cytokine production patterns by murine splenocytes stimulated with TGF- $\beta$ plus IL-6 were examined. As shown in Table 1, ST28 significantly $(P<0.01,(-)$ versus ST28) repressed IL-17 production (78\% reduction). On the other hand, ATCC 19258 failed to repress its production. Based on these results, ST28 was judged to be a suitable candidate strain for the treatment of Th17-mediated diseases, and its suppressive effects were further examined in vivo using DSS-induced colitis mice.

In regard to other cytokines than IL-17, the productions of all cytokines tested were changed by the stimulation with TGF- $\beta$ plus IL-6. Among them, IFN- $\gamma$ and TNF- $\alpha$ productions in the ST28 group were significantly higher than those in the ATCC 19258 group, and IL-2 and IL-4 productions were significantly lower. On the other hand, there were not significantly differences in IL-10 (anti-inflammatory cytokine) and IL-12 (proinflammatory cytokines) productions between the two groups.

3.3. S. thermophilus ST28 Suppressed Ex Vivo IL-17 Production. Acute colitis was induced in mice by exposure to $3.5 \%$ DSS for 5 days, which resulted in an increase in colon inflammation score compared to the control group (score, $2.5 \pm 1.5$ versus $0.5 \pm 0.3$ ) (Figure 2). However, oral treatment with ST28 ameliorated the inflammation (score, $1.0 \pm 0.4$ ). ATCC 19258 exerted the same ameliorative effect on the intestinal inflammation, but the effect was weaker (Figures 2(b) and 2(c)).

Next, LPLs from the mice was applied to ex vivo culture experiment (Figure 3). Although differences in IFN- $\gamma$ concentrations in LPLs culture were not prominent among four groups, LPLs from DSS colitis mice produced substantial level of IL-17. However, ST28 markedly suppressed IL-17 production from LPLs culture (86\% suppression), while ATCC 19258 failed to suppress IL-17 production.

From the analyses of mRNA expression in LPLs, it was revealed that the expression of both IL-17 and ROR$\gamma \mathrm{t}$, the master regulator of Th17, in LPLs from the DSSadministered group was drastically upregulated, compared to control mice (Supplementary Figure 1). Notably, ST28 markedly inhibited both expressions, which indicated that ST28 suppressed the differentiation of naïve T cells towards Th17 in LPLs. CD11c and CD86 were also found to be downregulated by the oral administration of ST28.

It was also confirmed by flow cytometry that $\mathrm{CD} 4^{+} \mathrm{IL} 17^{+}$ cells (Th17 cells) disappeared in LPLs by the oral administration of both ST28 and ATCC 19258 (Figure 4(a)). On the other hand, the effects of DSS and those of the administration of ST28 and ATCC 19258 on CD $11 \mathrm{c}^{+} \mathrm{CD} 86^{+}$ cells, inflammatory dendritic cells (DC), were unclear, although ATCC 19258 seemed to decrease the percentage of $\mathrm{CD} 11 \mathrm{c}^{+} \mathrm{CD} 86^{+}$cells. To clarify this point, we next evaluated the effect of ST28 on DC.

\subsection{S. thermophilus ST28 Suppressed Inflammatory Responses in BMDC. The effect of ST28 on LPS-matured BMDC was evaluated (Figure 5). LPS significantly induced IL-6 and IL-10 productions from BMDC. IL-10 production was not significantly decreased by the addition of ST28 and ATCC 19258. However, both ST28 and ATCC 19258 significantly suppressed IL-6 production. Accordingly, the percentage of CD86 cells, which is a hallmark of maturation [22], was also decreased by ST28 and ATCC 19258. Therefore, it is highly probable that ST28 suppressed Th17 cells via a DC- dependent mechanism.}

\section{Discussion}

In medical therapy for IBD, the modulation of immune responses and the regulation of intestinal inflammation is essential. Although the pathogenesis of IBD is complex, involving environmental, microbial, genetic, and immunological factors, several recent studies have shown that Th17 cells play a significant role in IBD pathology $[23,24]$. Here, we demonstrated that $S$. thermophilus ST28 repressed IL17 production in mouse splenocytes under Th17-skewed conditions in vitro (Table 1). Moreover, oral treatment of DSS-induced colitis mice with ST28 suppressed inflammatory Th17 cells in LPLs. Although S. thermophilus is not commonly considered a probiotic bacterium, the findings of the present study imply that this species would be useful in the treatment of IBD by suppressing exaggerated Th17 activity in inflamed intestines.

Th1/Th2 balance can influence the direction and outcomes of immune responses, due to the mutually antagonistic nature of Th1 and Th2 responses. In this regard, the modulating activity of probiotic and commensal bacteria on Th1/Th2 balance is commonly evaluated [3, 4]. Here, as IFN- $\gamma /$ IL- 4 production ratio in ST28 group was high even in Th17-skewed conditions (Table 1), it is expected that ST28 would suppress not only Th17, but also Th2 cells. This response would also affect IgE production in allergic conditions, because Th2 cells drive IgE class switching by allergen-stimulated B cells. Suppressing both Th2 and Th17 cells is desirable for the repression of IgE-mediated reaction, because these cell types are closely related with each other in allergic patients [25].

To confirm that ST28 also suppressed Th17 cells in vivo, acute colitis was induced by DSS treatment of mice. DSS induces acute inflammation and recruitment of immune cells, whose subsequent activation directly causes epithelial cell [19]. In DSS-induced colitis mice, which serve as a useful model of human IBD, ST28 administration improved inflammation score (Figure 1), repressed IL-17 production 
(Figure 3), and decreased Th17 cells (Figure 4) in LPLs isolated from inflamed intestines. Moreover, ST28 decreased the mRNA expressions of both ROR $y$ t and IL-17 in LPLs (Supplementary Figure 1). Considering all of the data, we conclude that ST28 suppresses Th17 differentiation in the DSS-induced colitis model. Although several lines of evidence indicate probiotic bacteria have beneficial effects on DSS colitis $[7,14,26]$, the mechanisms by which bacteria mitigate intestinal inflammation have not been fully clarified. We propose that ST28 ameliorates DSS-induced colitis in mice by suppression of inflammatory Th17 cells. It should be noted that even "heat-killed" bacteria could suppress IL17 production in this study. Therefore, some heat-resistant components of ST28 might exert Th17 supprressive activity. The influence of heating condition on this activity is also now evaluated.

The next question is that how ST28 and its responsible components suppress Th17 cells. It is generally accepted that bacterial components are recognized by members of the pattern recognition receptor family, such as Toll-like receptors (TLRs) [27]. In regard to the epithelial barrier, some studies have shown that the TLR2 ligand PCSK ameliorated TNF- $\alpha$-induced intestinal barrier impairment in the human epithelial Caco-2 cells [28, 29] and DSS colitis mice [29]. It is likely that the recognition of heat-resistant bacterial components by these kinds of receptors is also involved in the suppression of Th17 cells.

It has been recently suggested that CD86 plays a critical role in the initiation of T-cell responses including Th17 cells. For example, gene silencing of CD40, CD80, and CD86 protected collagen-induced arthritis, one of the Th17related inflammation [30]. In the effector $\mathrm{CD}^{+}{ }^{+} \mathrm{T}$-cell responses inducing antigen-induced arthritis, blockade of CD86 significantly suppressed IL-17 production in splenocytes, and CD86 enhanced disease severity by upregulating IL-17 production [31]. Also, high cell surface expression of CD86 was observed in patients with IBD [32] and in mice with $\mathrm{CD}^{+}$T-cell-induced colitis [33]. These findings lead us to examine the effect of ST28 on the expression of CD86. The mRNA expressions of CD86 and CD11c were drastically upregulated in LPLs from DSS colitis mice, but they were downregulated by the oral administration of ST28 (Supplementary Figure 1). ST28 also significantly decreased the percentage of $\mathrm{CD}^{+} 6^{+}$cells in matured BMDC (Figure 5). Collectively, it is highly probable that ST28 ameliorated DSS induced colitis by suppressing $\mathrm{CD} 86^{+} \mathrm{DC}$, which needs to be clarified in detail.

The function of IL-17 in intestinal inflammation has remained controversial [34]. It has been described that IL17 is involved in the pathogenesis of DSS-induced colitis in mice [16], and IL-17 receptor signaling plays a critical role in the development of trinitrobenzenesulfonic acid-induced acute colitis in mice [35], yet another report has suggested that IL-17 might offer an inhibitory role in the development of DSS-induced colitis [36]. O'Connor Jr. et al. [37] also demonstrated the data on a protective function for IL17 in T-cell-mediated intestinal inflammation. In addition, several recent studies suggest that Th1 and Th17 both govern inflammation [38]. For example, Buonocore et al. [39] have recently suggested that a novel innate lymphoid cell population accumulates in the inflamed colon and induces IL-17 and IFN- $\gamma$. Further investigations on Th17/Th1 in the intestinal inflammation are required to clarify the beneficial functions of ST28 on Th17-mediated diseases.

In conclusion, we have demonstrated that ST28 ameliorates intestinal inflammation in DSS-induced colitis mice at least partially through suppression of inflammatory Th17 cells. The use of probiotic bacteria in IBD therapy has been investigated in a number of clinical studies [40]. Although the efficacy of this treatment approach was demonstrated for ulcerative colitis [6], the results for Crohn's disease are not yet clear owing to conflicting results and a paucity of trials [40]. In any case, the majority of the demonstrated curative and palliative effects of probiotic bacteria appear to be mediated by modulation of the intestinal immune system. Clinical approaches using probiotics are appealing due to a lack of toxicity and patient desire for the use of natural physiological approaches to treating disease [40]. Since S. thermophilus has a long history of being safely consumed in yogurt, its further application to the treatment and/or prevention of Th17mediated diseases such as IBD is expected to be beneficial.

\section{References}

[1] C. K. Dotterud, O. Storrø, R. Johnsen, and T. Øien, "Probiotics in pregnant women to prevent allergic disease: a randomized, double-blind trial," British Journal of Dermatology, vol. 163, no. 3, pp. 616-623, 2010.

[2] Y. Nonaka, T. Izumo, F. Izumi et al., "Antiallergic effects of Lactobacillus pentosus strain S-PT84 mediated by modulation of Th1/Th2 immunobalance and induction of IL-10 production," International Archives of Allergy and Immunology, vol. 145, no. 3, pp. 249-257, 2008.

[3] N. Iwabuchi, N. Takahashi, J. Z. Xiao, K. Miyaji, and K. Iwatsuki, "In vitro Th1 cytokine-independent Th2 suppressive effects of bifidobacteria," Microbiology and Immunology, vol. 51, no. 7, pp. 649-660, 2007.

[4] L. E. M. Niers, H. M. Timmerman, G. T. Rijkers et al., "Identification of strong interleukin-10 inducing lactic acid bacteria which down-regulate T helper type 2 cytokines," Clinical and Experimental Allergy, vol. 35, no. 11, pp. 14811489, 2005.

[5] T. Von der Weid, C. Bulliard, and E. J. Schiffrin, "Induction by a lactic acid bacterium of a population of CD4+ T cells with low proliferative capacity that produce transforming growth factor $\beta$ and interleukin-10," Clinical and Diagnostic Laboratory Immunology, vol. 8, no. 4, pp. 695-701, 2001.

[6] Y. Takeda, H. Nakase, K. Namba et al., "Upregulation of Tbet and tight junction molecules by Bifidobactrium longum improves colonic inflammation of ulcerative colitis," Inflammatory Bowel Diseases, vol. 15, no. 11, pp. 1617-1618, 2009.

[7] R. B. Sartor, "Therapeutic manipulation of the enteric microflora in inflammatory bowel diseases: antibiotics, probiotics, and prebiotics," Gastroenterology, vol. 126, no. 6, pp. 1620-1633, 2004.

[8] S. Ulisse, P. Gionchetti, S. D'Alò et al., "Expression of cytokines, inducible nitric oxide synthase, and matrix metalloproteinases in pouchitis: effects of probiotic treatment," American Journal of Gastroenterology, vol. 96, no. 9, pp. 26912699, 2001. 
[9] L. Steinman, "A brief history of TH17, the first major revision in the $\mathrm{T} H 1 / \mathrm{TH} 2$ hypothesis of $\mathrm{T}$ cell-mediated tissue damage," Nature Medicine, vol. 13, no. 2, pp. 139-145, 2007.

[10] T. Korn, E. Bettelli, M. Oukka, and V. K. Kuchroo, "IL-17 and Th17 cells,” Annual Review of Immunology, vol. 27, pp. 485517, 2009.

[11] R. J. Xavier and D. K. Podolsky, "Unravelling the pathogenesis of inflammatory bowel disease," Nature, vol. 448, no. 7152, pp. 427-434, 2007.

[12] W. B. van den Berg and P. Miossec, "IL-17 as a future therapeutic target for rheumatoid arthritis," Nature Reviews Rheumatology, vol. 5, no. 10, pp. 549-553, 2009.

[13] Y. Nishitani, T. Tanoue, K. Yamada et al., "Lactococcus lactis subsp. cremoris FC alleviates symptoms of colitis induced by dextran sulfate sodium in mice," International Immunopharmacology, vol. 9, no. 12, pp. 1444-1451, 2009.

[14] S. Tanabe, Y. Kinuta, and Y. Saito, "Bifidobacterium infantis suppresses proinflammatory interleukin-17 production in murine splenocytes and dextran sodium sulfate-induced intestinal inflammation," International Journal of Molecular Medicine, vol. 22, no. 2, pp. 181-185, 2008.

[15] T. Ogita, Y. Tanii, H. Morita, T. Suzuki, and S. Tanabe, "Suppression of Th17 response by Streptococcus thermophilus ST28 through induction of IFN- $\gamma$," International Journal of Molecular Medicine, vol. 28, no. 5, pp. 817-822, 2011.

[16] R. Ito, M. Kita, M. Shin-Ya et al., "Involvement of IL-17A in the pathogenesis of DSS-induced colitis in mice," Biochemical and Biophysical Research Communications, vol. 377, no. 1, pp. 12-16, 2008.

[17] S. Tanabe and S. Hochi, "Oral administration of a galactooligosaccharide preparation inhibits development of atopic dermatitis-like skin lesions in $\mathrm{NC/Nga} \mathrm{mice,"} \mathrm{International}$ Journal of Molecular Medicine, vol. 25, no. 3, pp. 331-336, 2010.

[18] E. Miyauchi, H. Morita, and S. Tanabe, "Lactobacillus rhamnosus alleviates intestinal barrier dysfunction in part by increasing expression of zonula occludens- 1 and myosin lightchain kinase in vivo," Journal of Dairy Science, vol. 92, no. 6, pp. 2400-2408, 2009.

[19] B. Weigmann, I. Tubbe, D. Seidel, A. Nicolaev, C. Becker, and M. F. Neurath, "Isolation and subsequent analysis of murine lamina propria mononuclear cells from colonic tissue," Nature protocols, vol. 2, no. 10, pp. 2307-2311, 2007.

[20] T. L. Denning, Y. C. Wang, S. R. Patel, I. R. Williams, and B. Pulendran, "Lamina propria macrophages and dendritic cells differentially induce regulatory and interleukin 17-producing T cell responses," Nature Immunology, vol. 8, no. 10, pp. 10861094, 2007.

[21] R. D’Arienzo, F. Maurano, P. Lavermicocca, E. Ricca, and M. Rossi, "Modulation of the immune response by probiotic strains in a mouse model of gluten sensitivity," Cytokine, vol. 48, no. 3, pp. 254-259, 2009.

[22] J. Banchereau, F. Briere, C. Caux et al., "Immunobiology of dendritic cells," Annual Review of Immunology, vol. 18, pp. 767-811, 2000.

[23] R. J. Xavier and D. K. Podolsky, "Unravelling the pathogenesis of inflammatory bowel disease," Nature, vol. 448, no. 7152, pp. 427-434, 2007.

[24] Z. Hovhannisyan, J. Treatman, D. R. Littman, and L. Mayer, "Characterization of interleukin-17-producing regulatory $\mathrm{T}$ cells in inflamed intestinal mucosa from patients with inflammatory bowel diseases," Gastroenterology, vol. 140, no. 3, pp. 957-965, 2011.
[25] M. Milovanovic, G. Drozdenko, C. Weise, M. Babina, and M. Worm, "Interleukin-17A promotes IgE production in human B cells," Journal of Investigative Dermatology, vol. 130, no. 11, pp. 2621-2628, 2010.

[26] N. S. Nanda Kumar, R. Balamurugan, K. Jayakanthan, A. Pulimood, S. Pugazhendhi, and B. S. Ramakrishna, "Probiotic administration alters the gut flora and attenuates colitis in mice administered dextran sodium sulfate," Journal of Gastroenterology and Hepatology, vol. 23, no. 12, pp. 1834-1839, 2008.

[27] S. Uematsu and K. Fujimoto, "The innate immune system in the intestine," Microbiology and Immunology, vol. 54, no. 11, pp. 645-657, 2010.

[28] E. Miyauchi, H. Morita, J. Okuda, T. Sashihara, M. Shimizu, and S. Tanabe, "Cell wall fraction of Enterococcus hirae ameliorates TNF- $\alpha$-induced barrier impairment in the human epithelial tight junction," Letters in Applied Microbiology, vol. 46, no. 4, pp. 469-476, 2008.

[29] E. Cario, G. Gerken, and D. K. Podolsky, “Toll-like receptor 2 controls mucosal inflammation by regulating epithelial barrier function," Gastroenterology, vol. 132, no. 4, pp. 1359-1374, 2007.

[30] X. Zheng, M. Suzuki, T. E. Ichim et al., "Treatment of autoimmune arthritis using RNA interference-modulated dendritic cells," Journal of Immunology, vol. 184, no. 11, pp. 6457-6464, 2010.

[31] D. Odobasic, M. T. Leech, J. R. Xue, and S. R. Holdsworth, "Distinct in vivo roles of CD80 and CD86 in the effector T-cell responses inducing antigen-induced arthritis," Immunology, vol. 124, no. 4, pp. 503-513, 2008.

[32] S. J. Bell, R. Rigby, N. English et al., "Migration and maturation of human colonic dendritic cells," Journal of Immunology, vol. 166, no. 8, pp. 4958-4967, 2001.

[33] T. Krajina, F. Leithäuser, P. Möller, Z. Trobonjaca, and J. Reimann, "Colonic lamina propria dendritic cells in mice with $\mathrm{CD}^{+}{ }^{+} \mathrm{T}$ cell-induced colitis," European Journal of Immunology, vol. 33, no. 4, pp. 1073-1083, 2003.

[34] W. O’Connor Jr., M. Kamanaka, C. J. Booth et al., "A protective function for interleukin $17 \mathrm{~A}$ in $\mathrm{T}$ cell-mediated intestinal inflammation," Nature Immunology, vol. 10, no. 6, pp. 603609, 2009.

[35] Z. Zhang, M. Zheng, J. Bindas, P. Schwarzenberger, and J. K. Kolls, "Critical role of IL-17 receptor signaling in acute TNBSinduced colitis," Inflammatory Bowel Diseases, vol. 12, no. 5, pp. 382-388, 2006.

[36] A. Ogawa, A. Andoh, Y. Araki, T. Bamba, and Y. Fujiyama, "Neutralization of interleukin-17 aggravates dextran sulfate sodium-induced colitis in mice," Clinical Immunology, vol. 110, no. 1, pp. 55-62, 2004.

[37] W. O'Connor Jr., L. A. Zenewicz, and R. A. Flavell, "The dual nature of TH 17 cells: shifting the focus to function," Nature Immunology, vol. 11, no. 6, pp. 471-476, 2010.

[38] W. Strober, F. Zhang, A. Kitani, I. Fuss, and S. Fichtner-Feigl, "Proinflammatory cytokines underlying the inflammation of Crohn's disease," Current Opinion in Gastroenterology, vol. 26, no. 4, pp. 310-317, 2010.

[39] S. Buonocore, P. P. Ahern, H. H. Uhlig et al., "Innate lymphoid cells drive interleukin-23-dependent innate intestinal pathology," Nature, vol. 464, no. 7293, pp. 1371-1375, 2010.

[40] C. Hedin, K. Whelan, and J. O. Lindsay, "Evidence for the use of probiotics and prebiotics in inflammatory bowel disease: a review of clinical trials," Proceedings of the Nutrition Society, vol. 66, no. 3, pp. 307-315, 2007. 


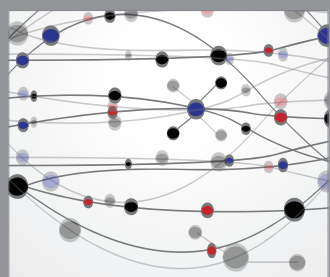

The Scientific World Journal
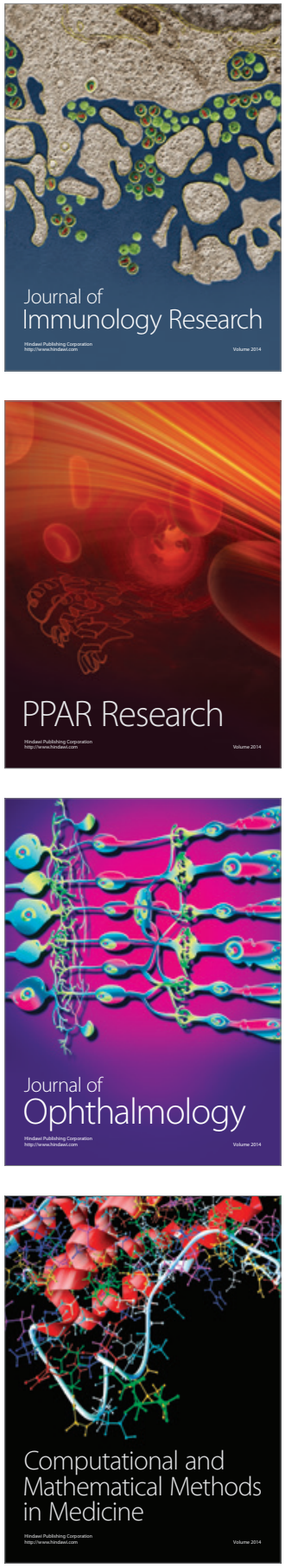

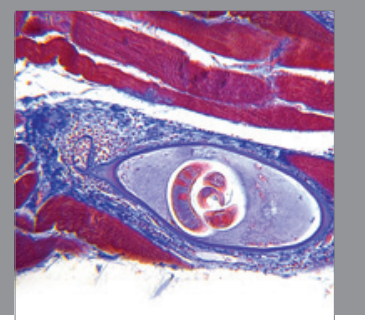

Gastroenterology

Research and Practice
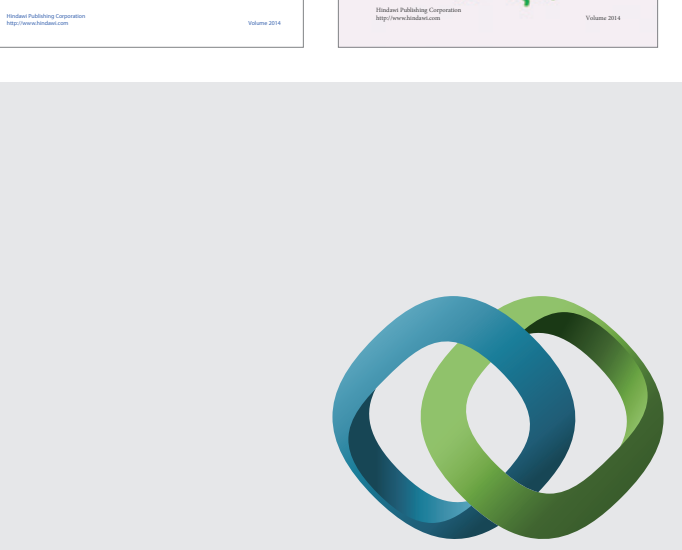

\section{Hindawi}

Submit your manuscripts at

http://www.hindawi.com
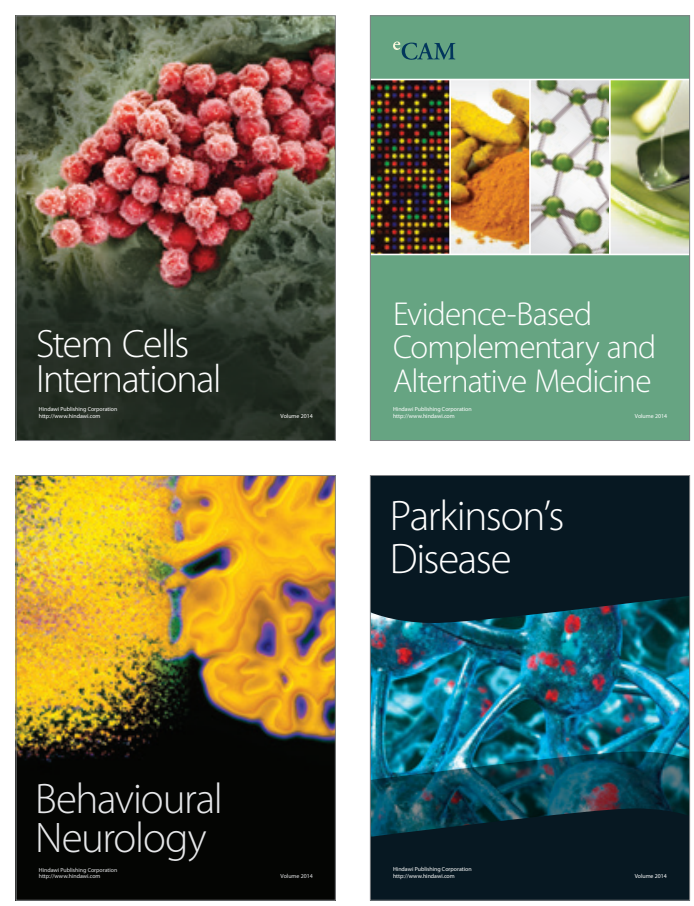

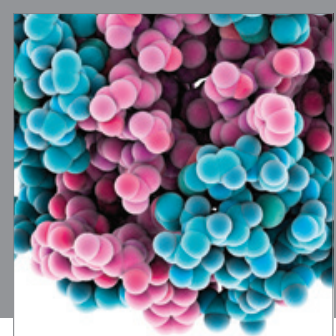

Journal of
Diabetes Research

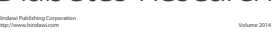

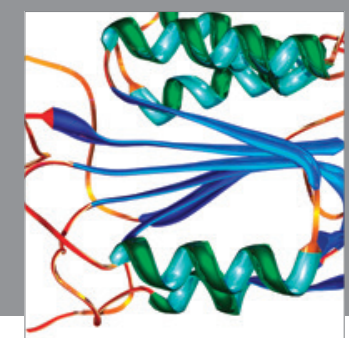

Disease Markers
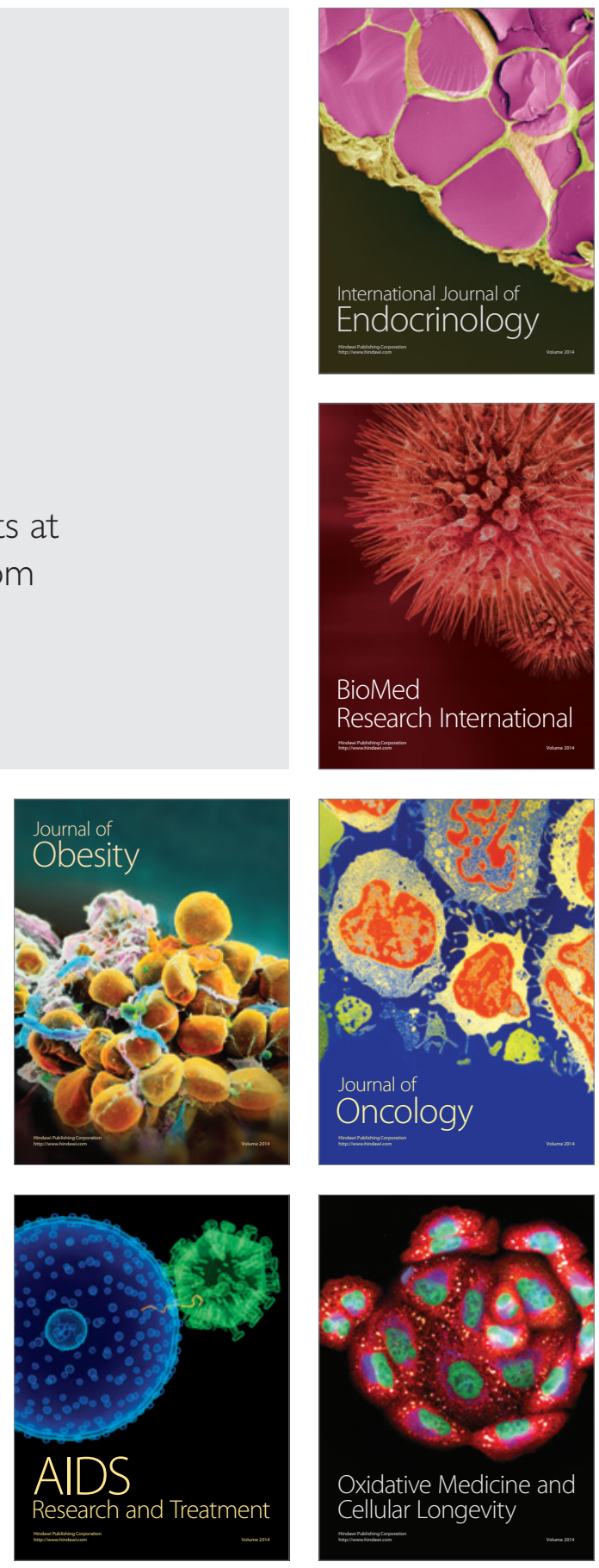